HUB-EP-97/21

LMU-HEP-97-5

SFB-375/164

hep-th/9704013

\title{
Instanton effects in string cosmology
}

\author{
Klaus Behrndt ${ }^{a}$, Stefan Förste ${ }^{b}$ and Stefan Schwager ${ }^{b}$ \\ a Humboldt-Universität, Institut für Physik \\ Invalidenstraße 110, 10115 Berlin, Germany \\ b Sektion Physik, Universität München \\ Theresienstraße 37, 80333 München, Germany
}

\begin{abstract}
We consider the gauge dyonic string solution of the $K 3$ compactified heterotic string theory in a four dimensional cosmological context. Since for this solution GreenSchwarz as well as Chern-Simons corrections have been taken into account it contains both world sheet and string loop corrections. The cosmological picture is obtained by rotating the world volume of the gauge dyonic string into two space like dimensions and compactifying those dimensions on a two torus. We compare the result with gauge neutral extreme and non-extreme cosmologies and find that the non-trivial Yang Mills background leads to a solution without any singularities whereas for trivial Yang-Mills backgrounds some of the fields become always singular at the big bang.
\end{abstract}

\footnotetext{
${ }^{1}$ e-mail: behrndt@qft2.physik.hu-berlin.de, Stefan.Foerste@physik.uni-muenchen.de, Stefan.Schwager@physik.uni-muenchen.de
} 


\section{Introduction}

Since string theory is supposed to describe physics at scales which are not reachable in any terrestrial laboratory cosmological models predicted by string theory might be the suitable candidates for facing this theory with nature. Therefore there has been quite some interest in studying cosmological vacua of string theory [1-25]. A major approach in studying string cosmology is based on the pre-big-bang scenario [3-6], (for a recent review containing additional references on the topic see [6]). In the pre-big-bang scenario two cosmological solutions related by scale factor duality are connected in a strong coupling region. Since one of the solution describes an accelerated expanding universe (inflationary branch) whereas the second solution corresponds to a decelerated expanding universe (FRW branch) this scenario has many realistic phenomenological implications. However, it suffers from some open questions. Because the strong coupling region is hardly accessible one outstanding problem is the description of the details how the two solutions are smoothly connected (graceful exit problem) 25].

The second track one can follow is to find more general cosmological string vacua, e.g. with non vanishing spatial curvature. Here, one can employ the considerable progress in the understanding of low-energy solutions of string theory which has been made during the last years. Hopefully, this approach will finally converge with the one described above. A better understanding of low-energy solutions of string theory was achieved by interpreting those solutions as intersections of the $D$-brane solutions of type II string theory. First one reinterpreted the known 4-d black holes as bound states of brane solution. But the procedure is quite general and can also be used to find new solutions in 4 dimensions, not only of 0brane type (black holes) but also of 1-brane (string) or (-1)-brane type (instanton). For a classification of all multiple intersections of $D$-branes see 26 .

In the present paper we are going to discuss 4-d cosmological solutions obtained by compactification of 6-d heterotic string solutions. The standard cosmology is described in terms of a Robertson-Walker metric

$$
d s^{2}=-d \tau^{2}+K^{2}(\tau) d \Omega_{3, k}
$$

where $K$ is the world radius and $d \Omega_{3, k}^{2}=d \chi^{2}+\sin ^{2}(\sqrt{k} \chi) / k\left(d \theta^{2}+\sin ^{2} \theta d \omega\right)$, which is the 3 -d spherical volume measure with the curvature $k=-1,0,1$. This metric ansatz takes into account that the universe is homogeneous and isotropic (as a good approximation). Trans- 
lated into the brane picture this means, that the Robertson-Walker space-time describes a (-1)-brane, i.e. the big bang is a point in the 4-d space-time.

Following this philosophy, there are two ways to obtain cosmological solutions from intersecting branes. The first one is to consider already a (-1)-brane in 10 dimensions and as second way one can consider the "standard" brane intersection and wraps the complete world volumes of the branes into the internal space. In order to be able to wrap the world volume of the brane one has to rotate the world volume such that the time coordinate is orthogonal to it. This can be done by performing a special analytic continuation (Wick-rotation) or by taking a non-extreme brane solution and going behind the outer horizon where the time and radius coordinates interchange.

In heterotic string theory, one has the fundamental string and NS-5-brane solution in 10d. Wrapping the 5-brane world volume into the internal space one gets immediately a cosmological solution in 4-d. Making this solution non-extremal and going behind the horizon one obtains a cosmological model that includes all possible values for the spatial curvature $k$ [10, 11]. Since the $N S-5$-brane is part of all superstring theories one can explore the type IIB $S$-duality in order to convert the brane into a $R R$-5-brane 18]. This way one can get some insight into strong coupling corrections to cosmological string vacua since $R R$ charged states are non perturbative states. A more systematic approach to cosmological solutions coming from any type of branes has been discussed in [21]. The field equations of any (non-extreme) brane solution, which shall be wrapped completely into the internal space can be reduced to 1-d field equations. In special cases one can solve these equations or use known solutions, e.g. in terms of Toda models [16]. In this procedure one has, however, to take into account that the intersections of extreme branes always yield a trivial Einstein frame metric [19]. Since in these models the big bang appears as the surface of the $D$-branes one can argue, that these branes could yield to a resolution of the big bang singularity [22].

In the present paper we will find that the inclusion of world sheet loop corrections $\left(\alpha^{\prime}\right)$ in addition to string loop corrections $\left(e^{\phi}\right)$ might lead to a completely non singular universe. A solution containing the two kinds of corrections is the gauge dyonic string constructed in [32]. This solution contains an $S U(2)$ instanton and thus Chern-Simons and Green-Schwarz corrections are non-trivial. Our aim in this paper is to investigate those "instanton effects" in a four dimensional cosmological context. Therefore, our starting point is a heterotic 6-d model, which corresponds in 10-d to a fundamental string lying inside an NS-5-brane (see figure 1). We will compactify this heterotic solution on a $K 3$ manifold yielding an $N=2$ 


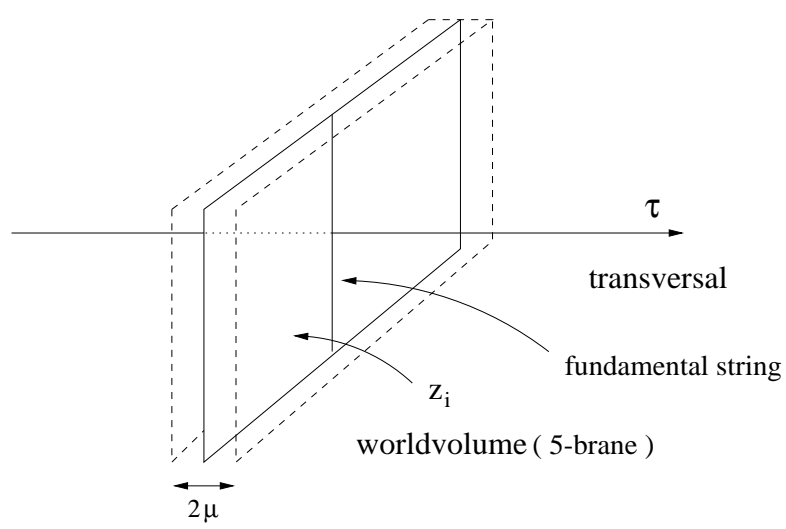

Figure 1: This figure shows a fundamental string lying in a NS-5-brane. The dashed line indicates the non-extreme version of this intersection. Note, there is a horizon only for $k=+1$. We have rotated this configuration so that the time $\tau$ is the transverse direction.

string cosmology in 4 dimensions after further compactification on $T^{2}$.

The paper is organized as follows. In the next section we will discuss the $K 3$ compactified heterotic string and describe the toroidal compactification down to four dimensions. In section 3 we study various six dimensional vacua describing (macroscopic) strings. By an analytic continuation we rotate the world volume of those strings into space-like directions and obtain the (-1)-brane configurations required for a cosmological interpretation. Section 4 deals with the resulting four dimensional cosmologies. We will first review known results for the instanton-free case and afterwards we will discuss the special effect of the YM instantons. We will also comment on the corresponding brane picture. Finally, we will summarize our results.

\section{The heterotic string theory on $K 3 \times T^{2}$}

In this section we will develop the general setting for compactifying the $K 3$ compactified heterotic string theory further down on $T^{2}$. The bosonic part of the six dimensional vacuum satisfies the following set of equations [27] $(M, N=0, \ldots, 5)$

$$
\begin{aligned}
\square \phi= & -\frac{1}{12} e^{-2 \phi} H^{2}+\frac{\alpha^{\prime}}{16} \sum_{\alpha}\left(v_{\alpha} e^{-\phi}-\tilde{v}_{\alpha} e^{\phi}\right) \operatorname{tr} F_{\alpha}^{2}, \\
R_{M N}= & \partial_{M} \phi \partial_{N} \phi+\frac{1}{4} e^{-2 \phi}\left(H_{M N}^{2}-\frac{1}{6} H^{2} G_{M N}\right) \\
& -\frac{\alpha^{\prime}}{4} \sum_{\alpha}\left(v_{\alpha} e^{-\phi}+\tilde{v}_{\alpha} e^{\phi}\right) \operatorname{tr}\left(F_{\alpha M N}^{2}-\frac{1}{8} F_{\alpha}^{2} G_{M N}\right),
\end{aligned}
$$




$$
\begin{gathered}
D_{M}\left(\left(v_{\alpha} e^{-\phi}+\tilde{v}_{\alpha} e^{\phi}\right) F_{\alpha}^{M N}\right)-\frac{1}{2} v_{\alpha} e^{-2 \phi} H_{P Q}^{N} F_{\alpha}^{P Q}-\frac{1}{2} \tilde{v}_{\alpha}{ }^{*} H_{P Q}^{N} F_{\alpha}^{P Q}=0, \\
d H=\frac{\alpha^{\prime}}{4} \sum_{\alpha} v_{\alpha} t r F_{\alpha} \wedge F_{\alpha}, \\
d \tilde{H}=\frac{\alpha^{\prime}}{4} \sum_{\alpha} \tilde{v}_{\alpha} t r F_{\alpha} \wedge F_{\alpha} .
\end{gathered}
$$

where $\tilde{H}=e^{-2 \phi *} H$. The solution we are going to consider has vanishing Lorentz ChernSimons forms and vanishing $R \wedge R$ and we did not include those contributions into the above set of equations. Terms with $v_{\alpha} F_{\alpha} \wedge F_{\alpha}$ are due to gauge Chern-Simons corrections whereas terms with $\tilde{v}_{\alpha} F_{\alpha} \wedge F_{\alpha}$ originate from the Green-Schwarz term. (Here, $\alpha$ labels an unbroken non Abelian subgroup of $E_{8} \times E_{8}$ or $S O(32)$ and we assume that the unbroken gauge symmetry is a direct product of non Abelian factors, only.) The values for $v$ and $\tilde{v}$ depend on the instanton embedding in the internal dimensions and can be found e.g. in appendix $\mathrm{B}$ of [28]. For the symmetric embedding of 12 instantons in each $E_{8}$ of the $E_{8} \times E_{8}$ string $v$ is always positive whereas the perturbative value for $\tilde{v}$ vanishes. It has been conjectured however that $\tilde{v}$ becomes equal to $v$ due to non perturbative effects [28, 29]. For the asymmetric instanton embedding and the $S O(32)$ string $\tilde{v}$ is negative and a phase transition occurs when the gauge kinetic term changes sign [28, 30, 31, 32].

The six dimensional vacua we are interested in fit into the following ansatz $(\mu, \nu=$ $0, \ldots, 3)$,

$$
d s_{6}^{2}=e^{-A(x)} \hat{g}_{\mu \nu}(x) d x^{\mu} d x^{\nu}+\frac{e^{A(x)}}{U_{2}}(d y+U d z)(d y+\bar{U} d z),
$$

where

$$
U=U(x)=U_{1}+i U_{2}
$$

is the complex structure of the torus we are going to compactify on. For the fields we choose the following ansatz,

$$
\begin{gathered}
\phi=\phi(x) \\
H_{y z \mu}=\partial_{\mu} b(x), \\
H_{\mu \nu \rho}=e^{2 \phi-2 A} \sqrt{\hat{g}} \epsilon_{\mu \nu \rho \lambda} \hat{g}^{\lambda \kappa} \partial_{\kappa} a \\
F_{\mu \nu}^{\alpha}=F_{\mu \nu}^{\alpha}(x)
\end{gathered}
$$

and the rest of the fields is zero.

Now, we are going to perform the dimensional reduction on a two torus with the coordinates $y, z$ at the level of the action. For simplicity we neglect Green-Schwarz terms 
$\left(B \wedge F_{\alpha} \wedge F_{\alpha}\right)$ at the moment and deduce their contribution in the end by general arguments. Since $F_{y z}=F_{y \nu}=F_{z \nu}=0$ those terms will give rise to $b \tilde{v}_{\alpha} F_{\alpha} \wedge F_{\alpha}$ contributions to the four dimensional Lagrangian. Without Green-Schwarz terms the six dimensional action is 9

$$
S_{6} \sim \int d^{6} x \sqrt{G_{6}}\left\{R_{6}-(\partial \phi)^{2}-\frac{e^{-2 \phi}}{12} H^{2}-\frac{\alpha^{\prime}}{8} \sum_{\alpha}\left(v_{\alpha} e^{-\phi}+\tilde{v}_{\alpha} e^{\phi}\right) \operatorname{tr} F_{\alpha}^{2}\right\},
$$

The ansatz (11) can be thought of as just using $H_{\mu \nu \rho}=H_{\mu \nu \rho}(x)$ and going to the dual axion $a$ in four dimensions. This is done by adding the Bianchi identity (5) to the action with a Lagrange multiplier $a$ and integrating out $H$. The Chern-Simons corrections will result in terms containing $a v_{\alpha} F_{\alpha} \wedge F_{\alpha}$. At the moment we drop also these contributions and reinstall them in the end together with the above neglected Green-Schwarz terms. The form of the metric (7) gives rise to the following expression for the scalar curvature, (scalar products, covariant derivatives are taken with respect to $\hat{g}$ ),

$$
R=e^{A}\left\{\hat{R}+\hat{\nabla}^{2} A+2 \frac{\partial U \partial \bar{U}}{(U-\bar{U})^{2}}-(\partial A)^{2}\right\} .
$$

In the compactified theory it is convenient to redefine fields according to

$$
\begin{aligned}
& A=\lambda-\varphi, \\
& \phi=\varphi+\lambda
\end{aligned}
$$

and to define the complex axion-dilaton field $S$ as

$$
S=S_{1}+i S_{2}=a+i e^{-2 \varphi}
$$

and the complex Kähler structure $T$ as

$$
T=T_{1}+i T_{2}=b+i e^{2 \lambda}
$$

Integrating (13) over the two torus (labeled by $y, z$ ) results in

$$
\begin{aligned}
S \sim & \int d^{4} x \sqrt{\hat{g}}\left\{\hat{R}+2 \frac{\partial U \partial \bar{U}}{(U-\bar{U})^{2}}+2 \frac{\partial S \partial \bar{S}}{(S-\bar{S})^{2}}+2 \frac{\partial T \partial \bar{T}}{(T-\bar{T})^{2}}\right. \\
& \left.-\frac{\alpha^{\prime}}{8} \sum_{\alpha}\left(v_{\alpha} S_{2}+\tilde{v}_{\alpha} T_{2}\right) t r F_{\alpha}^{2}\right\} .
\end{aligned}
$$

\footnotetext{
${ }^{2}$ This is for the convention that the universal sector of the effective string frame action is given by $S \sim \int \sqrt{G} e^{-2 \phi}\left[R+4(\partial \phi)^{2}-\frac{1}{12} H^{2}\right]$.
} 
Now, let us infer the Chern-Simons and Green-Schwarz corrections by using general arguments. The gravitational Green-Schwarz and Chern-Simons correction are of higher order in the derivatives and can be neglected at the considered order. In addition, for the vacuum we are going to study $R \wedge R$ vanishes identically. As discussed before the gauge field dependence of the neglected contributions will be of the form $\operatorname{tr} F \tilde{F}$ with $\tilde{F}$ being the Hodge dual of $F$ (in four dimensions). Supersymmetry requires holomorphic gauge couplings and that fixes the final expression of the reduced action to 33

$$
\begin{aligned}
S \sim & \int d^{4} x \sqrt{\hat{g}}\left\{\hat{R}+2 \frac{\partial U \partial \bar{U}}{(U-\bar{U})^{2}}+2 \frac{\partial S \partial \bar{S}}{(S-\bar{S})^{2}}+2 \frac{\partial T \partial \bar{T}}{(T-\bar{T})^{2}}\right. \\
& \left.-\frac{\alpha^{\prime}}{8} \sum_{\alpha} \operatorname{Im}\left[\left(v_{\alpha} S+\tilde{v}_{\alpha} T\right)\left(\operatorname{tr} F_{\alpha}^{2}-\frac{i}{2} \operatorname{tr} F_{\alpha} \tilde{F}_{\alpha}\right)\right]\right\} .
\end{aligned}
$$

These gauge field couplings follow also from an holomorphic prepotential as described e.g. in 34.

\section{The six dimensional solution}

In this section we will discuss the six dimensional vacuum which will lead us to a four dimensional cosmological solution upon toroidal compactification. First, we will discuss a string solution with a trivial Yang-Mills background and the analytic continuation giving the four dimensional cosmological interpretation. This will be done for the extreme and the nonextreme solution. In the extreme case we can switch on a non-trivial Yang-Mills background. That will be a modified form of the instanton solution discussed in [32]. The unbroken gauge symmetry in six dimensions is taken to be $S U(2)$. After an analytic continuation the gauge field will take values in the subset of $S L(2, C)$ that can be viewed as a Euclideanized version of $S L(2, \mathbb{R})$.

\subsection{Extreme and non-extreme vacuum with vanishing Yang-Mills background}

The non-extreme dyonic string solution has been constructed in 35 starting from the extreme solution of [36]. The issue how to obtain non-extreme solutions from extreme ones is also discussed in [37. For completeness we give the dyonic black string solution in six dimensions before performing the analytic continuation.

$$
d s^{2}=e^{A}\left(-d t^{2} e^{2 f}+d z^{2}\right)+e^{-A}\left(d r^{2} e^{-2 f}+r^{2} d \Omega_{3}^{2}\right)
$$


with

$$
\begin{gathered}
d \Omega_{3}^{2}=d \chi^{2}+\sin ^{2} \chi\left(d \theta^{2}+\sin ^{2} \theta d \omega^{2}\right), \\
e^{2 f}=1-\frac{\mu^{2}}{r^{2}} .
\end{gathered}
$$

For the rest of the fields one finds

$$
\begin{aligned}
& e^{2 \varphi}=1+\frac{\mu^{2}}{r^{2}} \sinh ^{2} \alpha \\
& e^{-2 \lambda}=1+\frac{\mu^{2}}{r^{2}} \sinh ^{2} \beta
\end{aligned}
$$

with $\varphi$ and $\lambda$ as in (15) and (16). The $H$ field is the sum of a contribution coupling to the electric (fundamental) string and a contribution coupling to the magnetic string,

$$
H=H_{e}+H_{m}
$$

with

$$
\begin{aligned}
& H_{e}=\operatorname{coth} \beta d e^{2 \lambda} \wedge d t \wedge d z, \\
& H_{m}=\operatorname{coth} \alpha r^{3} \partial_{r} e^{2 \varphi} \sin ^{2} \chi \sin \theta d \chi \wedge d \theta \wedge d \omega .
\end{aligned}
$$

The extreme solution is obtained by performing the limit $\mu \rightarrow 0, \alpha \rightarrow \infty$ and $\beta \rightarrow \infty$ such that

$$
\mu^{2} \sinh ^{2} \alpha \rightarrow P \quad, \quad \mu^{2} \sinh ^{2} \beta \rightarrow Q
$$

with $P$ and $Q$ finite. In our context another branch of the solution will be important. That branch is not considered in 35 since fields become singular at $r^{2}=\mu^{2} \cosh ^{2} \alpha$ and $r^{2}=\mu^{2} \cosh ^{2} \beta$. In the second solution one finds

$$
\begin{aligned}
& e^{2 \varphi}=1-\frac{\mu^{2}}{r^{2}} \cosh ^{2} \alpha \\
& e^{-2 \lambda}=1-\frac{\mu^{2}}{r^{2}} \cosh ^{2} \beta,
\end{aligned}
$$

and

$$
\begin{aligned}
& H_{e}=\tanh \beta d e^{2 \lambda} \wedge d t \wedge d z \\
& H_{m}=\tanh \alpha r^{3} \partial_{r} e^{2 \varphi} \sin ^{2} \chi \sin \theta d \chi \wedge d \theta \wedge d \omega .
\end{aligned}
$$

In the non-extreme case there are basically two options to obtain a solution serving our purpose of obtaining a four dimensional cosmological background. In order to interpret $t$ and $z$ as coordinates of an internal torus one needs to change the sign of the $t t$ component of the metric. In addition, a time-like coordinate in the compactified solution is required. A homogeneous and isotropic 4-d-time dependent solution is obtained by flipping the signature of the $r r$ metric component. For the non-extreme solution this happens when we take $r$ to 
be in the region between the inner and the outer horizon, i.e. $r^{2}<\mu^{2}$. The second option which we will consider in the rest of the paper is given by performing a Wick rotation

$$
\begin{gathered}
t=i y, \\
r=i \tau, \\
\chi \rightarrow i \chi .
\end{gathered}
$$

Note that the Wick rotation here and in the following does not change the signature of the 6-d theorie, it remains a Minkowskian theory. Therefore, the action as well as the equations of motion remain the same and the rotated solutions still solve eqs. (2) - (6), however, with a non-compact gauge group (see next section). The aim of this rotation is to convert the radius into a timlike direction and the time into a spatial direction. The continued solution reads

$$
\begin{gathered}
d s^{2}=e^{A}\left(d y^{2} e^{2 f}+d z^{2}\right)+e^{-A}\left(-d \tau^{2} e^{-2 f}+\tau^{2} d \Omega_{3,-1}^{2}\right), \\
d \Omega_{3,-1}^{2}=d \chi^{2}+\sinh ^{2} \chi\left(d \theta^{2}+\sin ^{2} \theta d \omega^{2}\right), \\
e^{2 f}=1+\frac{\mu^{2}}{\tau^{2}}, \\
e^{2 \varphi} \quad=1-\frac{\mu^{2}}{\tau^{2}} \sinh ^{2} \alpha, \\
e^{-2 \lambda}=1-\frac{\mu^{2}}{\tau^{2}} \sinh ^{2} \beta,
\end{gathered}
$$

and

$$
\begin{aligned}
& H_{e}=i \operatorname{coth} \beta d e^{2 \lambda} \wedge d y \wedge d z, \\
& H_{m}=i \operatorname{coth} \alpha \tau^{3} \partial_{\tau} e^{2 \varphi} \sinh ^{2} \chi \sin \theta d \chi \wedge d \theta \wedge d \omega,
\end{aligned}
$$

for the first branch (24), (26). Whereas the second branch (28), (29) continues to

$$
\begin{array}{ll}
e^{2 \varphi} & =1+\frac{\mu^{2}}{\tau^{2}} \cosh ^{2} \alpha, \\
e^{-2 \lambda} & =1+\frac{\mu^{2}}{\tau^{2}} \cosh ^{2} \beta,
\end{array}
$$

and

$$
\begin{aligned}
& H_{e}=i \tanh \beta d e^{2 \lambda} \wedge d y \wedge d z, \\
& H_{m}=i \tanh \alpha \tau^{3} \partial_{\tau} e^{2 \varphi} \sinh ^{2} \chi \sin \theta d \chi \wedge d \theta \wedge d \omega .
\end{aligned}
$$

Taking in addition now $\alpha, \beta$ to $i \alpha, i \beta$ gives real magnetic and electric charges. In that case both solutions are related by replacing $\alpha, \beta$ with $\frac{\pi}{2}-\alpha, \frac{\pi}{2}-\beta$ and the only singularity ist at $\tau=0$. However, we will be interested in performing the extreme limit and have therefore to stick to real $\alpha$ and $\beta$. In order to have a singularity only at $\tau=0$ (the position of the 
brane) w we have to take solution (38), (39) and encounter the problem of having imaginary charges in the continued solution. We will give a conjectural resolution of that problem in paragraph 3.3 .

\subsection{Extreme vacuum with non-trivial Yang-Mills background \& analytic con- tinuation}

Now, we will analytically continue the gauge dyonic string solution of [32]. For that purpose let us rederive their solution in a way that allows us to perform a "continuous Wick rotation". That means that we take as a metric ansatz

$$
d s_{6}^{2}=e^{A}\left(-q d t^{2}+d z^{2}\right)+e^{-A}\left(\frac{d r^{2}}{q}+r^{2} d \Omega_{3, k}^{2}\right),
$$

with the $S_{k}^{3}$ measure

$$
d \Omega_{3, k}^{2}=d \chi^{2}+\frac{\sin ^{2}(\sqrt{k} \chi)}{k}\left(d \theta^{2}+\sin ^{2} \theta d \omega^{2}\right),
$$

and $q, k$ are real constants. A "continuous Wick rotation" is performed by taking $q$ from positive values through zero to negative values. The constant $k$ will be fixed by the equations of motion. We take the unbroken gauge symmetry to be $S U(2)$ and the gauge field strength to be self dual with respect to

$$
d s_{4}^{2}=\frac{d r^{2}}{q}+r^{2} d \Omega_{3, k}^{2} .
$$

An $S_{k}^{3}$ symmetric ansatz for the gauge field is given by

$$
\mathcal{A}(r)=\gamma(r) \sqrt{q} g^{-1} d g
$$

with

$$
\begin{aligned}
g= & \cos \sqrt{k} \chi-i \cos \omega \sin \theta \sin \sqrt{k} \chi \sigma_{1}-i \sin \omega \sin \theta \sin \sqrt{k} \chi \sigma_{2} \\
& -i \cos \theta \sin \sqrt{k} \chi \sigma_{3} .
\end{aligned}
$$

The self duality condition leads to the following differential equation

$$
r \gamma^{\prime}=2 \gamma(1-\sqrt{q} \gamma) \sqrt{\frac{q}{k}}
$$

with the solution

$$
\gamma(r)=\frac{\exp \left\{\sqrt{\frac{q}{k}} \log \frac{r^{2}}{\rho^{2} \sqrt{q}}\right\}}{\sqrt{q} \exp \left\{\sqrt{\frac{q}{k}} \log \frac{r^{2}}{\rho^{2} \sqrt{q}}\right\}+1},
$$

\footnotetext{
${ }^{3}$ This requirement corresponds to our picture of rotating the world volume of the brane which should not lead to additional singularities.
} 
where $\rho^{2} \sqrt{q}$ is an integration constant. The ansatz for the $H$-field is taken to be

$$
\begin{gathered}
H_{\chi \theta \omega}=\sqrt{q} \frac{r^{3} \sin ^{2} \sqrt{k} \chi \sin \theta}{k} \partial_{r} e^{C}, \\
H_{i j r}=\sqrt{q} \epsilon_{i j} \partial_{r} e^{\tilde{C}} .
\end{gathered}
$$

The Yang-Mills equation (4) is then satisfied if we choose $C=\varphi$ and $\tilde{C}=\lambda$. The $H$-field equation (6) and the Bianchi identity (5) lead to

$$
\begin{aligned}
& \square_{0} e^{\phi-A}=\frac{e^{-2 A}}{8 q} v \alpha^{\prime} \operatorname{tr} F^{2}, \\
& \square_{0} e^{-\phi-A}=\frac{e^{-2 A}}{8 q} \tilde{v} \alpha^{\prime} \operatorname{tr} F^{2},
\end{aligned}
$$

where $\square_{0}=\frac{1}{r^{3}} \partial_{r}\left(r^{3} \partial_{r}\right)$ is the flat Laplacian. The rest of the equations of motions is automatically satisfied apart from the angular components of the Einstein equations (3) which provide the condition

$$
k=q .
$$

Finally, $e^{-2 A} \operatorname{tr} F^{2}$ is given by

$$
\frac{e^{-2 A}}{4} \operatorname{tr} F^{2}=-24 q \frac{\rho^{4}}{\left(r^{2}+\rho^{2}\right)^{4}}
$$

and with (49) and (50) we have just rederived the equations given in [32]. After a further numerical rescaling of the group generators we can copy their solution, f

$$
\begin{gathered}
e^{2 \varphi}=e^{\phi_{0}}+\frac{2 v \alpha^{\prime}\left(2 \rho^{2}+\tau^{2}\right)}{\left(\rho^{2}+\tau^{2}\right)^{2}}, \\
e^{-2 \lambda}=e^{-\phi_{0}}+\frac{2 \tilde{v} \alpha^{\prime}\left(2 \rho^{2}+\tau^{2}\right)}{\left(\rho^{2}+\tau^{2}\right)^{2}} .
\end{gathered}
$$

This rederivation is useful since it teaches us some interesting facts about the analytic continuation. At the first sight it seems to be quite disappointing that we obtained the constraint (51) since that implies that we will not be able to get cosmological solutions with a flat or a spherical three-space. On the other hand this condition is logical for the following reasons. When we send $q=k$ to zero the spatial $S^{3}$ decompactifies to a flat space and hence the instanton solution loses its topological meaning of mapping the spatial $S^{3}$ onto the $S U(2)-S^{3}$. Considering the Cartan-Killing metric $\operatorname{tr}\left(\sqrt{q} g^{-1} d g\right)^{2}$ one observes that it

\footnotetext{
${ }^{4}$ With (51) our metric ansatz and the one in 32] are connected by simple coordinate transformations.
} 
remains negative definite also after the analytic continuation to negative $q$. That is, after the analytic continuation the gauge field takes values in the subset of $S L(2, C)$ that can be viewed as an Euclideanized version of $S L(2, \mathbb{R})$. So, we continue also the gauge group from one real realization $(S U(2))$ to another one $\left(S L(2, \mathbb{R})_{E}\right)$. Topologically we continue from a $S^{3}$ group manifold to a set of group elements with $S_{-1}^{3}$ topology and for negative values of $q$ the "instanton" maps a pseudo-sphere in the target space on a pseudo-sphere in the group space. In the following we will call this also instanton and drop the quotation marks.

\subsection{Analytic continuation in the string-probe action}

In this paragraph we are going to study how the analytic continuation (rotating the world volume of the string) is seen from the view point of a probe string. Thereby we will give a possible resolution of the imaginary charge problem (39). Here, we will focus on the extreme solutions. Let us recall the (for the present discussion) relevant parts of the extreme solution. In the string frame the extreme 6 -d string solution is of the following form

$$
\begin{aligned}
& d s_{6}^{2}=e^{2 \lambda}\left(-d t^{2}+d z^{2}\right)+e^{2 \varphi} d x^{\mu} d x^{\mu}, \\
& H_{e}=d\left(e^{2 \lambda} d t \wedge d z\right),
\end{aligned}
$$

whereas the continuation is of the form

$$
\begin{aligned}
& d s_{6}^{2}=e^{2 \lambda}\left(d y^{2}+d z^{2}\right)+e^{2 \varphi}\left(-d \tau^{2}+\tau^{2} d \Omega_{3,-1}^{2}\right), \\
& H_{e}=i d\left(e^{2 \lambda} d y \wedge d z\right) .
\end{aligned}
$$

First, we consider a string-probe in the 6-d string-background. The probe action is

$$
S=-\frac{1}{4 \pi \alpha^{\prime}} \int d^{2} \sigma \sqrt{\operatorname{det}\left(-g_{M N} \partial X^{M} \partial X^{N}\right)}+\frac{1}{4 \pi \alpha^{\prime}} \int B^{(2)},
$$

where $M, N=0, \ldots, 5$ and $H=d B^{(2)}$. Gauge fixing the world sheet diffeomorphisms via, ( $\sigma^{i}$ are the Minkowskian world sheet parameters),

$$
t=\sigma^{0}, z=-\sigma^{1}, X^{\mu}=X^{\mu}\left(\sigma^{0}\right)
$$

and plugging (54) with $B^{(2)}=e^{2 \lambda} d t \wedge d z$ into (56) results in

$$
S=\frac{R}{8 \pi \alpha^{\prime}} \int d \sigma^{0} e^{2 \varphi} v^{2}+\mathcal{O}\left(v^{4}\right)
$$

with $v^{\mu}=\frac{d X^{\mu}}{d \sigma^{0}}$ being a four dimensional velocity vector and $R$ is the length of the string. (In difference to 38] we have no contribution from the string at rest since we included an 
additional constant into $B^{(2)}$.) The arising picture is that the string-probe is extended along the $z$-axis and follows geodesics with respect to

$$
d s^{2}=e^{2 \varphi} d X^{\mu} d X^{\mu}
$$

with changing time $\sigma^{0}$.

In addition to the analytical continuation in target space we perform also a continuation in the probe action, namely we rotate the world volume of the probe such that the time direction is orthogonal to it. This is done by a Wick-rotation

$$
\sigma^{0} \rightarrow i \sigma^{0}
$$

modifying the probe-action (56) to

$$
S=-i \frac{1}{4 \pi \alpha^{\prime}} \int d^{2} \sigma \sqrt{\operatorname{det}\left(g_{M N} \partial X^{M} \partial X^{N}\right)}+\frac{1}{4 \pi \alpha^{\prime}} \int B^{(2)} .
$$

Now, we choose as a gauge

$$
\begin{aligned}
& y=\sigma^{0}, \quad z=-\sigma^{1}, \tau=\tau\left(\sigma^{0}\right) \\
& \chi=\chi\left(\sigma^{0}\right), \quad \theta=\theta\left(\sigma^{0}\right), \omega=\omega\left(\sigma^{0}\right)
\end{aligned}
$$

and plug (55) into (61) with $B^{(2)}=i e^{2 \lambda} d y \wedge d z$. The result is

$$
S=\frac{R}{8 \pi \alpha^{\prime}} \int d\left(i \sigma^{0}\right) e^{2 \varphi}\left\{\left(\frac{d \tau}{d \sigma^{0}}\right)^{2}-\tau^{2}\left[\left(\frac{d \chi}{d \sigma^{0}}\right)^{2}+\sinh ^{2} \chi\left(\left(\frac{d \theta}{d \sigma^{0}}\right)^{2}+\sin ^{2} \theta\left(\frac{d \omega}{d \sigma^{0}}\right)^{2}\right)\right]\right\}
$$

So, the picture is that the string-probe was replaced by a "world sheet instanton" mapping the Euclidean world sheet onto the internal target space torus (with coordinates $y$ and $z$ ) and being localized in space-time. With changing Eucledian world sheet time $\sigma^{0}$ the locus of the "world sheet instanton" in the four dimensional space-time will move along geodesics with respect to

$$
d s^{2}=e^{2 \varphi}\left(-d \tau^{2}+\tau^{2} d \Omega_{3,-1}^{2}\right) .
$$

That is, when we probe the four dimensional space-time with those "world sheet instantons" we will not encounter problems due to imaginary electric and magnetic charges, (for magnetic charges one has to repeat the consideration for a heterotic dual string-probe leading to the same result with $e^{\varphi}$ replaced by $e^{-\lambda}$ ). Finally, we mention that it might be interesting to include the non-Abelian background into the probe-action. For that one needs to derive a sigma model for the heterotic $K 3$-compactified string with unbroken gauge group $S U(2)$. Theoretically one could do this by $K 3$-compactifying the sigma model of the ten dimensional heterotic string [39] in a suitable instanton background. In praxis however, we do not know how to do this and leave this question for future research. 


\section{The 4-d cosmology}

In this section we discuss the 4-d cosmological models obtained by rotating the 1-brane in the way described above. We will first recall the case for zero Yang-Mills-fields, i.e. the cosmologies related to the dyonic string solutions of the Neveu-Schwarz sector of string theories. Here we distinguish an extremal BPS solution and its non-extremal generalization. We will then discuss the model obtained from the six dimensional gauge dyonic string, where the charges are due to a single $S U(2)$-instanton Yang-Mills field in the transverse space.

\subsection{The cosmology from the non-extremal dyonic string}

Performing the rotation and the compactification within the non-extremal generalization of the BPS dyonic string [35] and changing to the string frame, we obtain the four dimensional solution

$$
\begin{aligned}
d s^{2} & =\left(1+\frac{\mu^{2}}{\tau^{2}} \cosh ^{2} \alpha\right)\left(-\frac{d \tau^{2}}{1+\frac{\mu^{2}}{\tau^{2}}}+\tau^{2} d \Omega_{-1}^{2}\right) \\
e^{2 \varphi} & =1+\frac{\mu^{2}}{\tau^{2}} \cosh ^{2} \alpha \\
e^{-2 \lambda} & =1+\frac{\mu^{2}}{\tau^{2}} \cosh ^{2} \beta .
\end{aligned}
$$

To discuss qualitatively the evolution of this open FRW-universe we transform the time coordinate by $\tau=e^{\eta}-\frac{\mu^{2}}{4} e^{-\eta}$, giving the metric

$$
d s^{2}=\left[\left(e^{\eta}-\frac{\mu^{2}}{4} e^{-\eta}\right)^{2}+\mu^{2} \cosh ^{2} \alpha\right]\left(-d \eta^{2}+d \Omega_{-1}^{2}\right)
$$

For $\eta \rightarrow \pm \infty$, the geometry approaches flat Minkowski space, while for finite $\eta$ the geometry is $R \times S_{-1}^{3}$. This metric therefore describes two flat Minkowski spaces connected by a whormhole (figure 2a). The space-like part of the whormhole has its smallest extension at $\eta_{0}=\ln \frac{\mu}{2}$, but is always nonzero. Let us determine whether matter can pass the wormhole in finite time. For massive particles at rest the corresponding proper time would be given by

$$
s=\int_{\eta_{0}-\eta^{\prime}}^{\eta_{0}+\eta^{\prime}} d \eta\left[\left(e^{\eta}-\frac{\mu^{2}}{4} e^{-\eta}\right)^{2}+\mu^{2} \cosh ^{2} \alpha\right]^{1 / 2} .
$$

Since the integrand is regular, the integral is finite for finite $\eta^{\prime}$. Thus the "big bang" at $\eta_{0}$ is only finitely far in the past or future, as seen from any point inside the wormhole. The dilaton and axion however become singular at $\eta_{0}$, which corresponds to $\tau=0$. 
$2 \mathrm{a}$

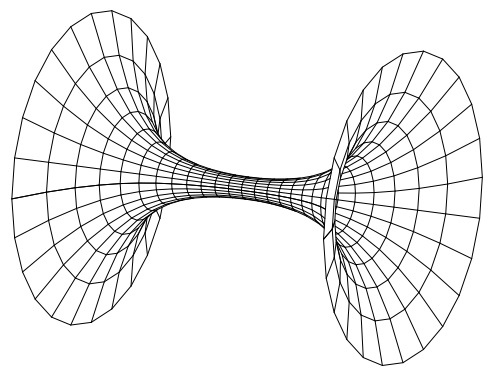

$2 \mathrm{c}$
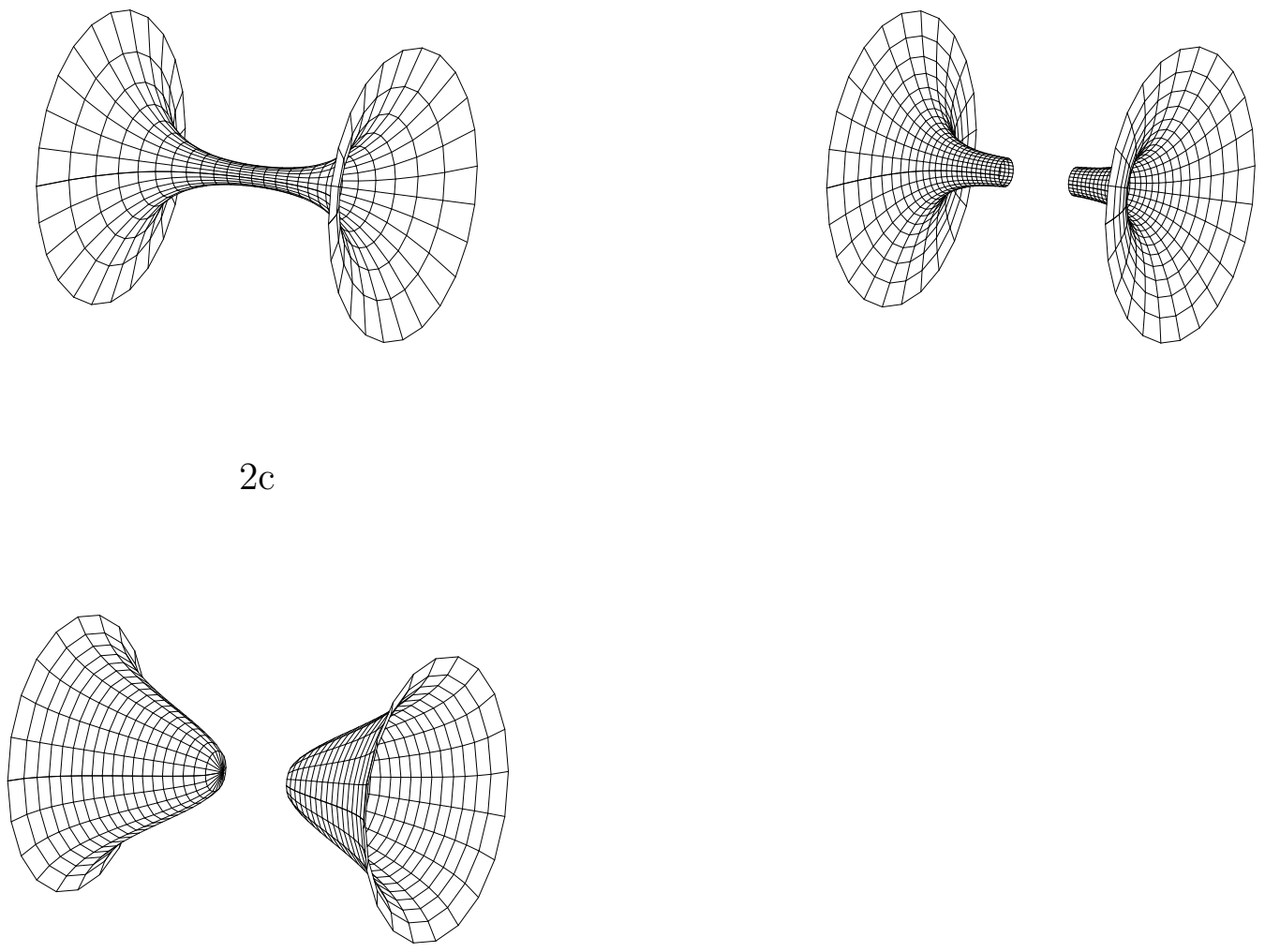

Figure 2: The cosmologies obtained from the nonextremal dyonic string (2a), its extremal BPS version (2b) and the BPS gauge dyonic string with a single $S U(2)$-instanton (2c).

\subsection{The cosmology from the extremal dyonic string}

The 6-d solution is the BPS dyonic solution of [36]. After performing the rotation and compactifying to four dimensions we obtain in the string frame

$$
\begin{aligned}
d s^{2} & =\left(1+\frac{P}{\tau^{2}}\right)\left(-d \tau^{2}+\tau^{2} d \Omega_{-1}^{2}\right), \\
e^{2 \varphi} & =1+\frac{P}{\tau^{2}}, \\
e^{-2 \lambda} & =1+\frac{Q}{\tau^{2}},
\end{aligned}
$$

with $Q$ the electric charge and $P$ the magnetic charge of the string. The solution corresponds to the extremal limit of (65), $\mu \rightarrow 0, \alpha, \beta \rightarrow \infty$ and $\mu^{2} \cosh ^{2} \alpha \rightarrow P, \mu^{2} \cosh ^{2} \beta \rightarrow Q$. The 
corresponding transformation of the time coordinate is now $\tau=e^{ \pm \eta}$, where the minus sign corresponds to the pre-big-bang phase, and we obtain for the extremal case

$$
d s^{2}=\left(e^{ \pm 2 \eta}+P\right)\left(-d \eta^{2}+d \Omega_{-1}^{2}\right)
$$

Considering the "+" branch, the geometry approaches again flat Minkowski space for $\eta \rightarrow \infty$, while for finite $\eta$ the geometry is $R \times S_{-1}^{3}$. The "big bang" however is now moved to $\eta=-\infty$ in the "+" branch and $\eta=\infty$ in the "-" branch, i.e. it is infinitely far in the past or future from each point in the throat. We can therefore think of performing the extremal limit as making the throat connecting the two asymptotic Minkowski spaces infinitely long and thereby physically separating the pre-big-bang from the post-big-bang phase. As in the nonextremal case, the dilaton and axion field diverge in the limit $\tau \rightarrow 0$, which corresponds to the big bang in both branches.

\subsection{The cosmology from the gauge dyonic string with $S U(2)$-instanton}

We now turn to the cosmology obtained from the gauge dyonic string of [32], where the charges of the string are produced by a single $S U(2)$-instanton. After performing the Wick rotation and compactifying to four dimensions we obtain the solution

$$
\begin{aligned}
d s^{2} & =\left(e^{2 \varphi_{0}}+2 \alpha^{\prime} v \frac{2 \rho^{2}+\tau^{2}}{\left(\rho^{2}+\tau^{2}\right)^{2}}\right)\left(-d \tau^{2}+\tau^{2} d \Omega_{-1}^{2}\right) \\
e^{2 \varphi} & =e^{2 \varphi_{0}}+2 \alpha^{\prime} v \frac{2 \rho^{2}+\tau^{2}}{\left(\rho^{2}+\tau^{2}\right)^{2}} \\
e^{-2 \lambda} & =e^{-2 \varphi_{0}}+2 \alpha^{\prime} \tilde{v} \frac{2 \rho^{2}+\tau^{2}}{\left(\rho^{2}+\tau^{2}\right)^{2}} .
\end{aligned}
$$

Again we transform the time coordinate, $\tau=e^{ \pm \eta}$, which gives the metric

$$
d s^{2}=e^{ \pm 2 \eta}\left(e^{2 \varphi_{0}}+2 \alpha^{\prime} v \frac{2 \rho^{2}+e^{ \pm 2 \eta}}{\left(\rho^{2}+e^{ \pm 2 \eta}\right)^{2}}\right)\left(-d \eta^{2}+d \Omega_{-1}^{2}\right) .
$$

Taking the limit $\eta \rightarrow \pm \infty$ in the "+" and "-" branch, respectively, we find again a decompactification of the $S_{-1}^{3}$ and therefore have asymptotic Minkowski spaces. However, the radius of the $S_{-1}^{3}$ shrinks to zero for $\eta \rightarrow \mp \infty$, indicating again a separation of the two branches (figure 2]c). We can get an upper bound for the proper time needed to reach the collapsing point from an arbitrary point

$$
s=\int_{-\infty}^{\eta^{\prime}} d s \leq e^{\eta^{\prime}} \sqrt{e^{2 \varphi_{0}}+\frac{2 \alpha^{\prime} v}{\rho^{2}}}
$$


(a)

(b)

(c)

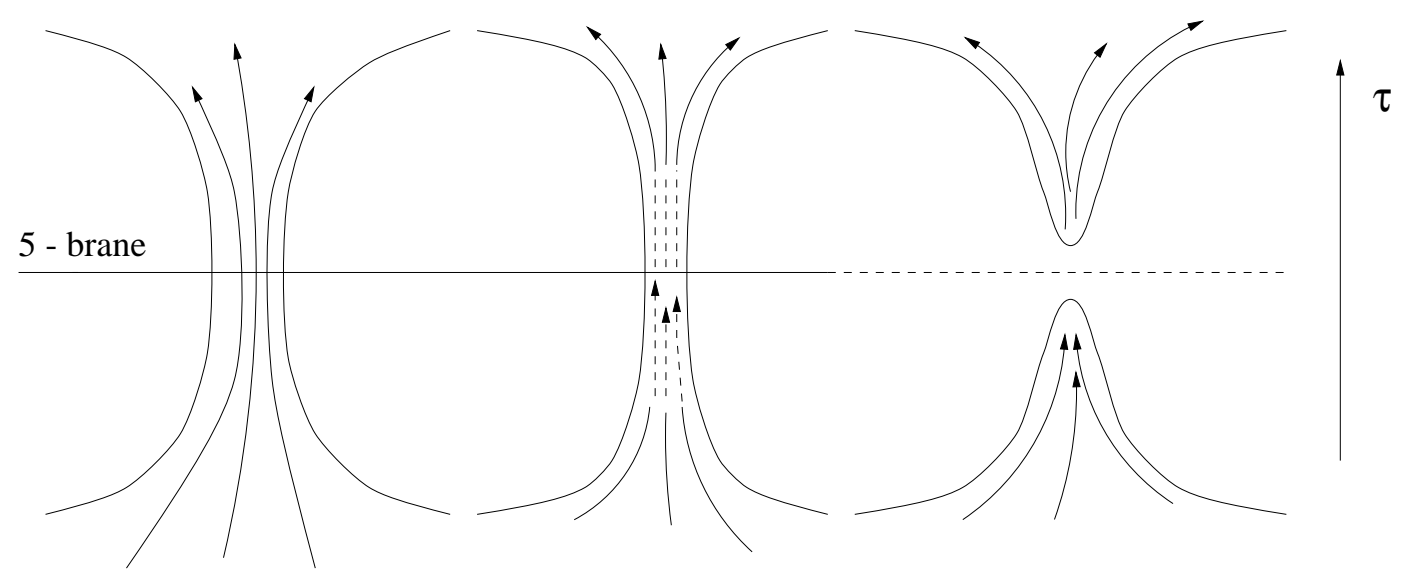

Figure 3: For the non-extreme 5-brane (a), matter can flow from one asymptotic flat region to the other. In the extreme limit (b), matter needs infinite time to reach or depart from the middle of the throat. So, both universes are causally disconnected. Taking an instanton source (c), results in a smooth "closure" of both regions.

which is finite for finite instanton size $\rho$. But at this point there is no singularity, all metric components as well as scalar fields stay finite here. We have a completely non-singular cosmological model. In the cases discussed before, the (string) metric was also finite, but the scalar fields were singular at $\tau=0$. At this point the internal torus collapsed and the string coupling (dilaton) diverged.

\subsection{The brane picture}

A main part of our cosmological model is the 5-brane. If we neglect for a moment the Yang Mills field, this 5-brane can be seen as a connection between two asymptotic flat regions (universes). But the second flat region is not on the other side of the 5-brane, instead one has to "go through the 5-brane" in order to reach it. This however is only possible for a non-extreme 5-brane, where matter needs a finite time to reach the other side, see eq. (67). Although the geometry is smooth (in the string-frame), the scalar fields and therefore also the gravitational coupling become singular in the "moment one touches the 5-brane". This point one could call the initial singularity at $\tau=0$. Since in our cosmological setting, we have rotated the world volume of the 5-brane in a way that the time is the transversal to the brane, matter can flow only in one direction. From the thermodynamical point of view this means, the configuration is not in an equilibrium as one would expect for a non-extremal 
solution.

Next, going to the extremal case means, that the length of the throat becomes infinite. In this case matter takes infinite time to reach or to depart from the 5-brane in the middle, see eq. (69). Thus, both asymptotic regions are causally disconnected, it is impossible to go "behind" the 5-brane.

Finally, turning on the Yang-Mills field, this hole in space time is closed by an instanton. In this case all fields behave smooth everywhere and the instanton can be seen as a cap that smoothly closes the 5-brane throat. It takes a finite time to reach the bottom. The scalar fields and also the gravitational coupling are bounded by the instanton size $\rho$, see eq. (70). Hence, the instanton keeps matter away from the original 5-brane and the two asymptotic regions are not only causally disconnected but also geometrically.

In this picture the fundamental string, that lies on the 5-brane seems to play no role. Really, turning on and off the electric charge has no influence on the 4-d geometry and neither on the 4-d dilaton. The world-volume of the fundamental string is mapped completely on the internal torus and therefore in 4- $\mathrm{d}$ we do not have anymore an electric charge, instead we can see it as topological charge that tells us how many times we wrapped the world volume. In the moment that one touches the 5-brane this internal torus shrinks to zero size indicating the singularity at this point. Again, turning on the YM instanton regularizes also this torus.

\section{Conclusions}

In the present paper we studied solutions of heterotic string theory corresponding to four dimensional cosmological solutions. The ten dimensional configuration is a fundamental string within a solitonic five brane. $K 3$ compactifying that background to six dimensions and wrapping the five brane around $K 3$ results in a dyonic string solution in six dimensions. As sources for those strings we considered delta function like sources and instantons. In the case of a delta function source extreme and non-extreme solutions are known [35, 36] whereas the background with an instanton source is known only in the extreme case [32]. We rotated the world volume such that the time is orthogonal to it and compactified the world volume on $T^{2}$ ending up with a four dimensional cosmological solution.

In the non-extreme solution with delta function source, (which now acts as a big bang singularity), we obtained two asymptotically flat regions connected by a wormhole. Matter 
can tunnel through the big bang singularity and would face singular background fields at the big bang. In the extreme limit of that solution matter takes infinite time to reach the singularity and the universe looks like a half throat with one asymptotically flat region. In the case where the instanton is the source of the six dimensional string we observed an asymptotically flat region at one end of the universe and a smoothly vanishing world radius at the other end. In the six dimensional model the finite instanton size corresponds to a spatially extended source for the string. After rotating the world volume of the string into space like directions this gets translated into a big bang source during a finite time interval resulting in a completely non singular model. It might be interesting to investigate whether these or similar effects can help to address the graceful exit problem of the pre-big-bang scenario [3] 6 .

\section{Acknowledgments}

We would like to thank Thomas Mohaupt and Stefan Theisen for useful discussions. S.F. and S.S. benefited from discussions with Debashis Ghoshal. They also acknowledge the hospitality of Institut für Physik at Humboldt University during a stay where part of the presented results was obtained. K.B. thanks Stefan Theisen for an invitation to Munich university.

The presented work is partly supported by the DFG through SFB 375-95 and TMR programs ERBFMX-CT96-0045 and ERBFMX-CT96-0090. K.B. and S.S. are supported by DFG, Deutsche Forschungsgemeinschaft. The work of S.F. is supported by GIF, German Israeli Foundation for scientific research. 


\section{References}

[1] I. Antoniadis, C. Bachas, J. Ellis and D.V. Nanopoulus, "An expanding universe in string theory" Nucl. Phys. B328 (1989) 117.

[2] A.A. Tseytlin, "Cosmological solutions with dilaton and maximally symmetric space in string theory", Int. J. Mod. Phys. D1 (1992) 223, hep-th/9203033.

[3] M. Gasperini and G. Veneziano, "Pre-Big Bang in String Cosmology", Astropart. Phys. 1 (1993) 317; hep-th/9211021. "Inflation, deflation and frame independence in string cosmology", Mod. Phys. Lett. A8 (1993) 3701, hep-th/9309023; "Dilaton production in string theory", Phys. Rev. D50 (1994) 2519, gr-qc/9403031.

[4] R. Brustein and G. Veneziano, "The graceful exit problem in string cosmology", Phys. Lett. B329 (1994) 429. hep-th/9403060.

[5] R. Brustein, M. Gasperini, M. Giovannini, V.F. Mukhanov and G. Veneziano, "Metric pertubations in dilaton driven inflation", Phys. Rev. D51 (1995) 6744, hep-th/9501066; G. Veneziano, "Inhomogenous Pre-Big Bang String Cosmology", hep-th/9703150.

[6] M. Gasperini, "Relic Dilatons in string cosmology", in "Proc. of the 12th Italian Conference on Gen. Rel. and Gravitational Physics" (Rome, September 1996), ed. by M. Bassan et al. (World Scientific, Singapore), gr-qc/9611059.

[7] C.R. Nappi and E. Witten, "A closed, expanding universe in string theory", Phys. Lett. B293 (1992) 309, hep-th/9206078.

[8] A. Giveon and A. Pasquinucci, "On cosmological string backgrounds with toroidal isometries", Phys. Lett. B294 (1992) 162, hep-th/9208076.

[9] D. Lüst, "Cosmological string backgrounds", Presented at 4th Hellenic School on Elementary Particle Physics, Corfu, Greece, 2-20 Sep 1992, hep-th/9303175.

[10] K. Behrndt and S. Förste, "Cosmological string solutions in four dimensions from 5d black holes", Phys. Lett. B320 (1994) 253, hep-th/9308131; “String Kaluza-Klein cosmology", Nucl. Phys. B430 (1994) 441, hep-th/9403179. 
[11] E.J. Copeland, A. Lahiri and D. Wands, "Low-energy effective string cosmology", Phys. Rev. D50 (1994) 4868, hep-th/9406216; "String cosmology with a time dependent antisymmetric tensor", Phys. Rev. D51 (1995) 1569, hep-th/9410136.

[12] K. Behrndt and T. Burwick, "Towards quantum cosmology without singularities", Phys. Rev. D50 (1995) 1295, hep-th/9407039.

[13] J. Levin, "Inflation from extra dimensions", Phys. Lett. B343 (1995) 69, $\mathrm{gr-qc} / 9411041$.

[14] R. Easther, K. Maeda and D. Wands, "Tree level string cosmology", Phys. Rev. D53 (1996) 4247, hep-th/9509074.

[15] I. Antoniadis, J. Rizos and K. Tamvakis, "Singularity free cosmological solutions of the superstring effective action", Nucl. Phys. B415(1994) 497, hep-th/9305025; R. Easther and K. Maeda, "One loop superstring cosmology and the nonsingular universe", Phys. Rev. D54 (1996) 7252, hep-th/9605173.

[16] N. Kaloper, "Stringy Toda Cosmologies", Phys. Rev. D55 (1997) 3394, hep-th/9609087.

[17] H. Lü, S. Mukherji and C.N. Pope and K.W. Xu, "Cosmological solutions in string theories", hep-th/9610107.

[18] R. Poppe and S. Schwager, "String Kaluza-Klein cosmologies with RR fields", Phys. Lett. B393 (1997) 51, hep-th/9610166.

[19] A.A. Tseytlin, "On the structure of composite black p-brane configurations and related black holes", hep-th/9611111.

[20] E.J. Copeland, R. Easther and D. Wands, "Vacuum fluctuations in axion-dilaton cosmologies", hep-th/9701082.

[21] A. Lukas, B.A. Ovrut and D. Waldram, "Cosmological solutions in type II string theory", Phys. Lett. B393 (1997) 65, hep-th/9608195; "String and M-theory cosmological solutions with Ramond forms", hep-th/9610238; "Stabilizing dilaton and moduli vacua in string and $M$ theory", hep-th/9611204.

[22] F. Larsen and F. Wilczek, "Resolution of cosmological singularities", hep-th/9610252. 
[23] H. Lü, S. Mukherji and C.N. Pope, "From p-branes to cosmology", hep-th/9612224.

[24] S. Kalyana Rama, "Can string theory avoid cosmological singularities?", hep-th/9701154.

[25] R. Brustein and R. Madden, "Graceful exit and energy conditions in string cosmology", hep-th/9702043.

[26] E. Bergshoeff, M. de Roo, E. Eyras, B. Janssen and J.P. van der Schaar, "Mutliple intersections of D-branes and M-branes", hep-th/9612095.

[27] A. Sagnotti, "A note on the Green-Schwarz mechanism in open string theory", Phys. Lett. B294 (1992), hep-th/9210127.

[28] M.J. Duff, R. Minasian and E. Witten, "Evidence for heterotic/heterotic duality", Nucl. Phys. B465 (1996) 413, hep-th/9601036.

[29] M. Berkooz, R. Leigh, J. Polchinski, J. Schwarz, N. Seiberg and E. Witten, "Anomalies, Dualities and Topology of $D=6 N=1$ superstring vacua", Nucl. Phys. B475 (1996) 115 , hep-th/9605184.

[30] D.R. Morrison and C. Vafa, "Compactifications of F-theory on Calabi-Yau threefolds 1 \&3 2", Nucl. Phys. B473 (1996) 74, hep-th/9602114 \& Nucl. Phys. B476 (1996) 437, hep-th/9603116.

[31] G. Aldazabal, A. Font, L.E. Ibáñez and F. Quevedo, "Heterotic/heterotic duality in $D=6, D=4 "$, Phys. Lett. B380 (1996) 33, hep-th/9602097.

[32] M.J. Duff, H. Lü and C.N. Pope, "Heterotic phase transitions and singularities of the gauge dyonic string", Phys. Lett. B378 (1996) 101, hep-th/960303307.

[33] J. Wess and J. Bagger, "Supersymmetry and Supergravity", Princeton University Press (1983).

[34] G. Lopes Cardoso, G. Curio and D. Lüst, "Perturbative couplings and modular forms in $N=2$ string models with a Wilson line", hep-th/9608154.

[35] M.J. Duff, H. Lü and C.N. Pope, "The black branes of M-theory", Phys. Lett. B382 (1996) 73, hep-th/9604052. 
[36] M.J. Duff, S. Ferrara, R.R. Khuri and J. Rahmfeld, "Supersymmetry and dual string solitons", Phys. Lett. B356 (1995) 479, hep-th/9506057.

[37] M. Cvetič and A.A. Tseytlin, "Nonextreme black holes from nonextreme intersecting M-branes", Nucl. Phys. B478 (1996) 181, hep-th/9606033.

[38] M. Douglas, J. Polchinski and A. Strominger, "Probing five-dimensional black holes with D-branes", hep-th/9703031.

[39] A. Sen, "Equations of motion for the Heterotic String Theory from the conformal Invariance of the Sigma Model", Phys. Rev. Lett. 18 (1985) 1846; "Heterotic string in arbitrary background field", Phys. Rev. D32 (1985) 2102. 